\title{
Workshop/conference report
}

\section{Myalgic Encephalomyelitis/Chronic Fatigue Syndrome (ME/CFS) program and Interdisciplinary Research Symposium on Disabling Fatigue in Chronic Illness}

\author{
E. Stein, MD (1); M. MacQuarrie, BSc MRP LLB (2)
}

\section{Background}

This program at the University of Calgary was the first comprehensive program on myalgic encephalomyelitis/chronic fatigue syndrome (ME/CFS) held in Canada. There were three parts: a continuing medical education program for physicians, a research symposium on fatiguing illnesses and a public lecture. ME/CFS is one cause of disabling fatigue. ME/CFS alone is a serious medical condition that affects between $150000^{1}$ and $340000^{2}$ Canadians. The core symptoms have been described by Fukuda et al. $^{3}$ and were further refined in the Canadian Consensus Guidelines for the Diagnosis and Treatment of ME/CFS. ${ }^{4}$

\section{Program}

The physician program was divided into two segments: Part I (October 24, 2008) covered the diagnosis and assessment of patients with ME/CFS and Part II (November 7, 2008) covered clinical management.

A full-day interdisciplinary research symposium on disabling fatigue in chronic illness took place on November 8, 2008, in a mix of plenary and concurrent sessions. This symposium was organized to assist in both the development of a collaborative research agenda to understand disabling fatigue in chronic disease as well as in the knowledge transfer process among health care professionals, researchers and the Canadian public. Disabling fatigue exists in numerous chronic conditions, including autoimmune disorders, chronic infection, chronic liver disease, pulmonary and heart disease, ME/CFS, overtraining and stress syndromes, some psychiatric conditions and fatigue due to unknown causes.

The plenary sessions included Karin Olson, $\mathrm{RN}, \mathrm{PhD}$, University of Alberta (Conceptual Model of Fatigue: The Edmonton Fatigue Framework); Leonard Jason, PhD, DePaul University (Epidemiology and Case Definition of ME/CFS); Nancy Klimas, MD, University of Miami (Biological Markers in Disabling Fatigue); Bryan Kolb, PhD, University of Lethbridge (Neuroplasticity and Implications for Disabling Fatigue); and a video of Gerry Thomas, with a patient perspective.

Dr. Olson presented the Edmonton Fatigue Framework (EFF), a proposed etiological model of fatigue based on 15 years of research with five populations experiencing fatigue: those with cancer, depression and ME/CFS, as well as shift-workers and recreational runners. In this model, fatigue (subtyped as tiredness, fatigue and exhaustion) is considered a behavioural marker of the inability to adapt to stress and is secondary to changes in muscle endurance, sleep quality, cognitive function, dietary intake and other factors. ${ }^{5}$

Dr. Klimas, an internationally renowned research immunologist and clinician, profiled current work, including dynamic modelling using an exercise stressor model, showing that one must stress a subject with ME/CFS to get reliable differences versus controls. Mathematical modelling by Gordon Broderick, PhD (Computational Biology, University of Alberta and one of Dr. Klimas' team) is identifying which biomarkers could serve as a diagnostic test for ME/CFS. ${ }^{6}$

Dr. Jason spoke about the definition, prevalence and social impact of ME/ CFS and, in the concurrent session, about a four-arm, non-pharmacological intervention study in ME/CFS. Both of his presentations underscored the importance of properly identifying and subtyping ME/ CFS patients. Not every ME/CFS patient reacts to treatment in the same way.

Dr. Bryan Kolb (Director of the Canadian Centre for Behavioural Neuroscience at the University of Lethbridge and author of the classical textbook, Fundamentals of Human Neuropsychology $y^{7}$ reviewed the literature on brain plasticity and implications for ME/CFS. He made the

Author References

1 Private practice, Calgary, $A B$, Canada

2 Myalgic Encephalomyelitis Association of Ontario, Toronto, ON, Canada and member of the National ME/FM Action Network

Submitted by Ellie Stein, MD, (Chair, Program Committee) on behalf of the Planning Committee: Drs. Terrie Brandon (Family Medicine, Calgary); Brian Maclntosh

(Kinesiology, University of Calgary); Karin Olson (Nursing, University of Alberta); Steve Simpson (Medicine, University of Calgary); Elaine Stapon (Family Medicine, Calgary);

and Ms. Glenda Wong (Department of CME, University of Calgary).

Correspondence: Ellie Stein, MD, 4523-16A St. SW Calgary, AB, T2T 4L8, Tel.: 403-287-9941, Fax: 403-287-9958, Email: espc@shaw.ca 
provocative hypothesis that the increased prefrontal volume post "effective cognitive behavioural therapy" reported by de Lange et al. ${ }^{8}$ may have been due to the impact of the therapy on depression rather than ME/ CFS. Many symptoms, including depression and stress, correlate with structural changes in the prefrontal cortex.

The concurrent sessions were also filled with some very stimulating findings, including:

- Bruce Dick, PhD (Departments of Anesthesiology and Pain Medicine and Psychiatry, University of Alberta), presented work on cognitive function in fibromyalgia. ${ }^{9}$ He reported that the spatial span test was the most difficult task for people with fibromyalgia and that cognitive function did not improve in the short term with pain intervention. However, patients on long-term opiate treatment did better on cognitive tests overall than those who were not on opiates.

- Patrick Neary, PhD (Kinesology and Health Studies, University of Regina), presented his data on prefrontal cortical oxygenation, as measured by nearinfrared spectrophotometry during exercise to exhaustion. ${ }^{10}$ There was no difference at rest between the ME/CFS and control groups, but with exercise, both the total haemoglobin and oxygenated haemoglobin were reduced in the ME/CFS group. He has shown in unpublished work that oxygen flow to the brain is slow to recover when patients with ME/CFS stand up. This reinforces the need for provocation testing in ME/CFS.

- Carey Johnson, MD (private practice, Calgary), presented his observations that approximately $50 \%$ of patients with Erlers Danlos (ED) syndrome have clinical features meeting the Canadian Consensus Guidelines for the Diagnosis and Treatment of ME/CFS; the other $50 \%$ have the typical features of connective tissue disorder, but not chronic fatigue, sleep disorder, pain or sensory sensitivity. He has a study in progress to identify genetic markers for this subgroup of ED patients.
- Neil Skjodt, MD (Medical Director, Edmonton Sleep Institute; Director of Research, Canadian Sleep Institute), noted that the sleep irregularities in $\mathrm{ME} / \mathrm{CFS}$ were, for various reasons, not getting specialized attention. He suggested a more appropriate sleep assessment protocol for these patients.

Other thought-provoking and informative presentations were given by:

- Denise Adams, BSc (PhD candidate, University of Alberta), on Traditional Chinese Medicine for the Treatment of Chronic Fatigue: A Systematic Review;

- Brian MacIntosh, PhD (Kinesology, University of Calgary), on Measuring Peripheral vs. Central Fatigue;

- Lynn Marshall, MD (Environmental Health Clinic, Women's College Hospital, Toronto), on Functional Impairment in an Environmental Clinic Sample;

- Kathleen Pierson, MD, PhD (Department of Psychiatry, University of Calgary), on Measuring Fatigue in Early Psychosis;

- Steve Simpson, MD, FRCP(C) (Psychiatry, University of Calgary; Consulting Psychiatrist, Tom Baker Cancer Centre), on the Management of Cancer Fatigue;

- Mark Swain, MD, FRCP(C) (Professor of Medicine, University of Calgary), on Disabling Fatigue in Inflammatory Disorders; and

- Mark van Ness, PhD, Staci Stevens, MSc, and Kylie Kumasaka, PhD candidate (Pacific Fatigue Laboratory, University of the Pacific, California), on Metabolic Dysfunction in ME/CFS.

The public lecture on November 9, 2008 featured Nancy Klimas, MD, giving an update on ME/CFS research, Alison Bested, $\mathrm{MD}, \mathrm{FRCP}(\mathrm{C})$ (private practice, Toronto), providing clinical pearls ${ }^{11}$ and a tag team of Stevens, van Ness and Kumasaka presenting on Exercise Tolerance in ME/CFS.
Podcasts (i.e. audio and slides only) of the ME/CFS continuing medical education program and the public lecture are currently available free of charge. To view the podcasts go to: http://podcast. med.ucalgary.ca/groups/cfs/weblog/. The research day is not a podcast, although handouts from many of the presentations are available on the University of Calgary Continuing Medical Education site, at www.cme.calgary.ca.

\section{Conclusion}

There were many coinciding concepts from various presenters in different fields, specifically, the interrelation of all body systems and the need to take an interdisciplinary approach to understanding ME/CFS and disabling fatigue in chronic illness. The opportunity for so many disciplines to gather and benefit from "crosstalking" and the fertilization of ideas was immense, and new ideas and collaborations are ongoing. The objective of the symposium to develop a collaborative research agenda was ambitious. Meeting this objective will take time, effort and funding.

\section{Acknowledgements}

This program was co-endorsed by the University of Calgary, Faculty of Medicine, and the University of Alberta, Faculty of Medicine and Dentistry. It was supported by Alberta Health and Wellness, the Public Health Agency of Canada and the Canadian Institutes of Health Research. The ME-FM Society of Alberta was a major sponsor. Other sponsors (in alphabetical order) were Bayda Ludwar Law Firm, Cambrian Drug Mart, Ferring Pharmaceuticals, Genuine Health, Metagenics, Pfizer Canada, Script Pharmacy and Varsity Natural Health Center. The podcasts are available thanks to sponsorship from the Alison Hunter Memorial Foundation (www.ahmf.org). 


\section{References}

1. Jason LA, Richman JA, Rademaker F, et al. A community-based study of chronic fatigue syndrome. Arch Intern Med. 1999; 159(18):2129-37.

2. Park J, Knudson S. Medically unexplained physical symptoms. Health Rep. 2007; 18:45-9.

3. Fukuda K, Straus SE, Hickie I, Sharpe MC, Dobbins JG, Komaroff A. The chronic fatigue syndrome: a comprehensive approach to its definition and study. Ann Intern Med. 1994;121:953-9.

4. Carruthers BM, Jain AK, DeMeirleir K et al. Myalgic Encephalomyelitis/Chronic Fatigue Syndrome: clinical working case definition, diagnostic and treatment protocols - a consensus document. J Chronic Fatigue Syndr. 2003;11(1):7-115.

5. Olson K, Turner AR, Courneya KS, et al. Possible links between behavioural and psychological indices of tiredness, fatigue, and exhaustion in advanced cancer. Support Care Cancer. 2008;16(3):241-9.

6. Broderick G, Craddock RC, Whistler T, Taylor R, Klimas N, Unger ER. Identifying illness parameters in fatiguing syndromes using classical projection methods. Pharmacogenomics. 2006;7(3):407-19.

7. Kolb B, Whishaw IQ. Fundamentals of human neuropsychology. 6th ed. New York: Freeman-Worth; 2008. 763 p.

8. de Lange FP, Koers A, Kalkman JS, et al. Increase in prefrontal cortical volume following cognitive behavioural therapy in patients with chronic fatigue syndrome. Brain. 2008;131:2172-80.

9. Dick BD, Verrier MJ, Harker KT, Rashiq S. Disruption of cognitive function in Fibromyalgia Syndrome. Pain. 2008; 139(3):610-6.
10. Neary JP, Roberts AD, Leavins N, Harrison MF, Croll JC, Sexsmith JR. Prefrontal cortex oxygenation during incremental exercise in chronic fatigue syndrome. Clin Physiol Funct Imaging. 2008;28(6):364-72.

11. Bested, AC, Logan AC. Hope and help for chronic fatigue syndrome and fibromyalgia. 2nd ed. Nashville (TN): Cumberland House; 2008. 267 p. 
